See discussions, stats, and author profiles for this publication at: https://www.researchgate.net/publication/11249399

\title{
Can physical trauma cause breast cancer?
}

Article in European Journal of Cancer Prevention · June 2002

DOI: 10.1097/00008469-200206000-00014 - Source: PubMed

5 authors, including:

James A Morris

University Hospitals Of Morecambe Bay NHS Foundation Trust

119 PUBLICATIONS 2,695 CITATIONS

SEE PROFILE

Some of the authors of this publication are also working on these related projects:

Disease prevention View project

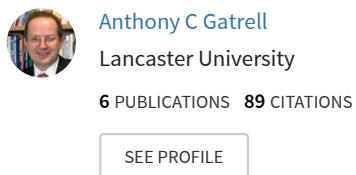




\title{
PILOT STUDY
}

\section{Can physical trauma cause breast cancer?}

\author{
J E Rigby ${ }^{1}$, J A Morris ${ }^{2}$, J Lavelle ${ }^{2}$, M Stewart ${ }^{2}$, A C Gatrell ${ }^{1}$ \\ (Received 25 November 2001; accepted 14 December 2001)
}

The objective of this study is to explore the effect of lifestyle on the risk of invasive breast carcinoma in women aged 50-65 years. A case-control study using a questionnaire and a semi-structured interview. Cases $(n=67)$ and controls $(n=134)$ were closely matched on known risk factors for breast cancer including age, family history, age at menarche, parity, age at first birth and menopausal status. Controls were chosen from a pool of 5600 women who attended for breast screening and filled in a questionnaire giving details to allow matching with cases. The study took place at the North Lancashire Breast Screening Service. Women were aged 50-65 years and presented with breast cancer or attended for breast screening.

Women with breast carcinoma were more likely to report physical trauma to the breast in the previous 5 years than were the controls (odds ratio (OR) 3.3, 95\% confidence interval (CI) $1.3-10.8, P<0.0001$ ). There were no significant differences in a wide range of other lifestyle indicators including factors relevant to social class, education, residence, smoking and alcohol consumption. In conclusion, recall bias is an unlikely explanation for these results in view of the nature and severity of physical trauma. Models of epithelial cell generation indicate that a causal link between physical trauma and cancer is plausible. A latent interval between cancer onset and presentation of under 5 years is also plausible. The most likely explanation of the findings is that physical trauma can cause breast cancer.

(C) 2002 Lippincott Williams \& Wilkins.

Key words: Breast cancer, physical trauma, trauma.

\section{Introduction}

In the United Kingdom, breast cancer is the most common cause of death for women in the 35-55 year age group. Incidence increases with age, particularly between the ages of 40 and 55, after which the increase is more gradual (Parkin et al., 1992). The aetiology is complex, but in addition to age, a number of risk factors have been identified, largely associated with reproductive history. Late age at first birth (MacMahon et al., 1970), nulliparity (Peeters et al., 1994), early age at menarche with late age at menopause (Brinton et al., 1988) and family history are all clearly implicated, but are explanatory factors for less than $50 \%$ of the incidence (Madigan et al., 1995). The epidemiology has been comprehensively reviewed by Kelsey (1993), Hulka and Stark (1995), and Lipworth (1995).

Changes in incidence of breast cancer amongst migrant groups towards those of their country of adoption (Buell, 1973) suggest that environmental factors may be relevant. Recent research has emphasized the importance of the intra-uterine environment upon the subsequent development of a number of adult diseases (Barker, 1992). Trichopoulos (1990) has suggested that breast cancer might originate in utero and this is supported by mathematical modelling of mutation in proliferating cell clones (Morris, 1991).

This study was designed as a case-control investigation of the possible influence of early life and later life events on the subsequent development of breast cancer.

\section{Subjects and methods}

The study was conducted within the area of operation of the North Lancashire Breast Screening Service, which covers Lancaster, Blackpool and Preston. The

${ }^{1}$ Department of Geography, Lancaster University, Lancaster LA1 4YB, UK. ${ }^{2}$ Royal Lancaster Infirmary, Ashton Road, Lancaster LA1 4RP, UK. Correspondence to: J A Morris. Fax: (+44) 1524583798. 
service offers routine screening by mammography, at 3 yearly intervals, to women in the 50-65 year age range.

The cases available to the study were women in the screening age range of 50-65 years, with a diagnosis of invasive breast carcinoma confirmed by biopsy. The study period ran from June 1996 to June 1998. The computer at the screening unit was interrogated periodically to produce a list of women with a malignant biopsy result. The appropriate breast care nurse was then contacted and she ascertained whether the patient was prepared to cooperate with the study. Eighty-two cases of breast cancer were identified and 67 were recruited to the study. The response rate was $81.7 \%$. Ten women had become ill as a result of treatment and were not asked to participate, one woman was not asked because her husband was seriously ill. Two women had moved out of the study area, two agreed to participate but subsequently declined to be interviewed. Thus, 15 cases were lost from the original 82 identified.

Controls were available from the 20000 women who attended for routine screening. At each screening session women were invited to participate in the study and to fill in a short questionnaire giving details of date of birth, age at menarche and menopause, parity and family history of breast cancer. Not all women who attended for screening were asked to fill in the questionnaire because of logistic problems, mainly related to lack of time at the screening site. Five thousand six hundred completed forms were obtained, however, and this was over $70 \%$ of those asked. For each case two controls were chosen from this pool of 5600 women. The aim was to match cases and controls to within 2 years of age, age of menarche, and age at first birth of parous. This was achieved for all but 11 of the 134 controls, where one of these three criteria had to be relaxed slightly. Cases and controls were also matched for menopausal status, family history of breast cancer and parity. Eight cases $(11.9 \%)$ reported their mother or sister (or both) having had breast cancer and 16 appropriate controls were found. Eight cases were nulliparous and appropriate controls were available. Cases and controls who indicated that they were adopted were not included in the study. From the original selections, seven controls declined to participate, with a further six controls having moved house. Replacements were therefore found.

The data were collected in a semi-structured interview whereby a questionnaire was completed to ascertain details of life-course events, exploring residential, occupational and reproductive histories, along with lifestyle indicators of smoking, alcohol consumption and stress. The final section of the questionnaire asked for the respondent's own thoughts concerning breast cancer, and issues identified were explored in subsequent interviews. Initial contact of cases and controls was by telephone, to arrange a date and time for the interview. The questionnaire was sent in advance to give the respondent some time to familiarize herself with the sensitive nature of some of the questions, and also to seek information from other members of their families. All the interviews were conducted by the same (female) researcher in the respondents' homes, with the exception of four interviews conducted at the place of work.

Following the interview with a case, a database of the potential controls was searched to identify those who matched on the risk factors discussed above. When more than two controls were identified those who had been screened the most recently were chosen. Controls were then contacted and interviewed. If, following the interview, it was found that a control no longer matched the case, a further control was sought. This occurred most commonly where the search had been for a nulliparous women, but on interview it transpired that the control had become pregnant but had not carried to term. Eight control interviews were thereby discarded.

The issue of physical trauma to the breast was raised by the first case interviewed, thus enabling this to be explored with all the other respondents. The index case recalled blunt trauma to the breast, which subsequently developed cancer, occurring within a period of 5 years prior to mammography. All other cases and controls were asked about physical trauma to the breast within this time period (i.e. 5 years prior to the interview). We did not have precise criteria for type or severity of trauma. Instead we recorded every example that the women remembered and regarded as significant.

\section{Results}

The controls were more likely to have breastfed their babies (of the parous cases $56 \%$ reported some experience of breastfeeding, compared with $63 \%$ of controls); they had achieved higher educational qualifications $(67 \%$ of cases had no qualifications compared with $46 \%$ of controls); they were less likely to have smoked $(60 \%$ of cases and $52 \%$ of controls had smoked at some stage); they drank less alcohol (25\% of cases and $7 \%$ of controls consumed more 
Table 1. The number of cases and controls reporting physical trauma to the breast in a 5-year interval prior to interview

\begin{tabular}{lcc}
\hline Nature of trauma & $\begin{array}{l}\text { Number of } \\
\text { cases }\end{array}$ & $\begin{array}{l}\text { Number of } \\
\text { controls }\end{array}$ \\
\hline Fall, injuring chest: & & \\
$\quad$ From bicycle & 2 & 0 \\
Down stairs & 2 & 3 \\
Other & 10 & 4 \\
Fall, possible injury to chest & 6 & 7 \\
Kicks, blows, knocks & 12 & 0 \\
Car accident & 3 & 1 \\
Horse bite & 0 & 1 \\
Total & $35(52 \%)$ & $16(12 \%)$ \\
\hline
\end{tabular}

than 14 units of alcohol per week). None of these differences reached statistical significance.

The cases reported significantly more physical trauma to the breast in the 5 years prior to screening than did the controls (Table 1). The recorded incidents were remembered clearly, by both cases and controls, as painful and distressing events. The recorded injuries in cases involved either both breasts or, if unilateral, the breast that subsequently developed cancer. The data were analysed using the approach for matched triples with dichotomous data as outlined by Breslow and Day (1980). This analysis gave an odds ratio of $3.3,95 \%$ confidence interval (CI) $1.3-10.8(P<0.0001)$.

\section{Discussion}

A strength of the current study is that it proved possible to match cases and controls for known risk factors. These include age, family history of breast cancer and factors related to reproductive history such as age at menarche, age at first birth, parity and menopausal status. A limitation is that although controls were considered to be clear of breast cancer at the time of screening the length of time for data collection meant that controls might not be contacted as matches for up to 18 months after they were screened. Additionally, although there was no reason to suspect selection bias amongst the cases, the pool of potential controls was very much a self-selecting group in that not all women who attended for screening volunteered to participate, and indeed not all eligible women attend for breast screening.

The main finding was a strong statistical association between reported trauma to the breast and the subsequent development, in a relatively short period, of invasive breast carcinoma. This is unlikely to be a result of selection bias as the cases and controls did not demonstrate any significant differences on a wide range of other lifestyle factors. There are, however, two major obstacles to accepting this association as a causal link. One is the possibility of recall bias and the other is the question of biological plausibility.

Recall bias is an important consideration. Women who have a positive diagnosis of breast cancer are likely to spend time thinking about what might have caused the disease and there is a possibility that they will remember events that the controls have forgotten. The breasts, however, are very sensitive parts of the body and it is intrinsically unlikely that the controls would have forgotten the type and severity of physical trauma that the cases have recorded in this study. For example, 12 of the cases, but none of the controls, remembered kicks, blows and knocks to the breast, some of which were the result of physical abuse. During the interview care was taken to give all the women time to think about the issue so that if trauma had occurred and was remembered it would be recalled. It is also important to note that the confidential nature of the personal interview was considered sufficiently supportive for respondents to volunteer information relating to physical abuse as one form of trauma.

A causal link between trauma and breast cancer in an interval under 5 years seems at first sight implausible and is certainly contrary to conventional concepts in carcinogenesis. The conventional view of epithelial cell generation is that stem cells divide asymmetrically, with one daughter cell becoming the new stem cell and the other undergoing a series of divisions to generate mature differentiated epithelial cells, which have a short life before they are shed. The stem cells then divide once more and the process is repeated. The most comprehensive set of kinetic data on epithelial cell generation is derived from work on the intestinal crypts of the small and large intestine (Potten and Loeffler, 1990). According to conventional models the stem cells in the crypts of the small intestine divide every few days and an individual stem cell in a 70-year-old person could be up to 5000 divisions from the zygote. In this process mutations will gradually accumulate in the stem cell genome and if a stem cell acquires a particular set of mutations it will proliferate out of control to cause cancer. There is no reason to believe that trauma increases mutagenesis and it is difficult to imagine how a single episode of trauma could lead to a significant increase in cancer risk in a short interval of time. Trauma could interfere with the blood supply, cause the release of stimulatory cytokines, or disrupt areas 
of ductal carcinoma-in situ. This could accelerate the growth and modify the time of presentation of established tumours, but to go from no malignancy to malignancy in less than 5 years is implausible if the conventional models are correct.

There is a different model of epithelial generation, however, which leads to a radically different prediction of the relationship between trauma and cancer (Morris, 1994, 1999). There are approximately $10^{14}$ human cells in an adult, each day $10^{12}$ mitoses occur and in a human lifetime approximately $10^{16}$ cells are produced in total. All of these cells are derived from a single cell, the zygote. If a zygote undergoes 60 successive divisions then $2^{60}$ cells would be produced and since $2^{60}=10^{18}$ this is enough for at least a 1000 years of human life. Thus, it is possible, in theory, to produce all the cells in a human lifetime with no cell even in extreme old age, more than 60 divisions from the zygote. This number is far less than in the conventional model in which stem cells can be over 5000 divisions from the zygote by 70 years of age. Limiting the number of cell generations to 60 leads to a vast reduction in the expected incidence of cancer (Morris, 1999) but demands precise control of the stem cell hierarchy. If control of the stem cell hierarchy is lost or the hierarchy is disrupted by factors such as trauma the risk of cancer will rise. In order to limit the number of cell generations to 60 it is necessary to produce a linear hierarchy of stem cells in which the more junior members, those furthest from the zygote, divide more frequently and the more senior members divide less frequently. The result is that mature epithelial cells are produced by junior stem cells and the junior stem cell pool is replenished by the division of senior stem cells. This model is described in detail elsewhere (Morris, 1994). Potentially malignant cells will arise among the junior members of the hierarchy, but they will be shed as long as the hierarchy is maintained. If the hierarchy is disrupted by events such as trauma the more junior stem cells could take over the hierarchy and the risk of cancer would rise. If the junior stem cell, which took over the hierarchy had already acquired the mutations for malignancy it could produce in a relatively short time a clinically detectable cancer. If the cells divide weekly a $1 \mathrm{~kg}$ mass would develop within a year. If the cells divide monthly a $1 \mathrm{~kg}$ mass would develop within 3 years. If the tumour doubling time was daily for the first 15 generations, weekly for the next 15, monthly for the next five and then 3 monthly thereafter; a $1 \mathrm{~kg}$ tumour mass with an observed doubling time of 3 months would arise within 2 years.
It is possible to devise models of epithelial cell generation that lie between the two extremes of a regularly cycling stem cell, which in old age is over 5000 divisions from the zygote, and a model in which no cell is more than 60 divisions from the zygote. All the intermediate models require some form of stem cell hierarchy with precise schedules of cell division. In all the intermediate models if trauma disrupts the hierarchy the incidence of cancer will rise and the latent interval to cancer presentation could be short. The arguments for and against the various models are presented elsewhere (Morris, 1999). None of the models has universal support but the rate at which telomeres shorten in humans with cell division argues against the extreme version of the conventional model. Thus a stem cell hierarchy of some form is required and consequently the concept that trauma can disrupt the hierarchy and increase the incidence of cancer is biologically plausible.

It is surprising that, although patients with cancer commonly report a history of trauma, there has been little systematic study of the possibility of a causal link. There is some evidence of a link between trauma and brain tumours (Inskip et al., 1998) and of a link between trauma and testicular cancer (UK Testicular Cancer Study Group, 1994). Case-control studies undertaken by the UK Testicular Cancer Study Group found testicular trauma at least 2 years prior to diagnosis associated with an odds ratio of $2(95 \%$ CI 1.54-2.61). In the testicular study attempts were made to reduce the problem of recall bias by confirming reports of trauma with the mother or with the casualty records.

The best way to eliminate recall bias is to perform a prospective study. This could be done with the breast screening population but up to 20000 women would need to be questioned about trauma prior to screening for the study to have sufficient statistical power. A study of this size would require information to be obtained by questionnaire rather than by interview and there is a risk that sensitive information would not be divulged. It is necessary therefore to make a judgement on the basis of information gained in retrospect as in this study. Contrary to the conventional view a causal link between physical trauma and breast cancer is biologically plausible and is the most likely explanation for our results.

\section{References}

Barker DJP (1992). Fetal and infant origins of adult disease. London $B M J$. 
Breslow NE, Day NE (1980). Statistical Methods in Cancer Research, Vol 1: The Analysis Of Case-Control Studies. IARC, Lyon.

Brinton LA, Schairer C, Hoover RN, Fraumeni JF (1988). Menstrual factors and risk of breast cancer. Cancer Invest 6: 245-54.

Buell P (1973). Changing incidence of breast cancer in JapaneseAmerican women. J Natl Cancer Inst 51: 1479-83.

Hulka BS, Stark AT (1995). Breast cancer: cause and prevention. Lancet 346: 883-7.

Inskip PD, Mellemkjaer L, Gridley G, Olsen JH (1998). Incidence of intracranial tumors following hospitalization for head injuries (Denmark). Cancer Causes Control 9: 109-16.

Kelsey JL (1993). Breast cancer. Epidemiol Rev 15: 7-16.

Lipworth L (1995). Epidemiology of breast cancer. Eur J Cancer Prev 4: 7-30.

MacMahon P, Cole P, Lin TM, et al. (1970). Age at first birth and breast cancer risk. Bull WHO 43: 209-21.

Madigan MP, Ziegler RG, Benichou J, Byrne C, Hoover RN (1995). Proportion of breast cancer cases in the US explained by well-established risk factors. J Natl Cancer Inst 87: 1681-95.

Morris JA (1990). The spontaneous mutation rate in retinoblastoma. J Clin Pathol 43: 496-8.

Morris JA (1991). A mutational model of carcinogenesis. Anticancer Res 11: 1725-30.

Morris JA (1994). Stem cell kinetics and intestinal cancer. Anticancer Res 14: 2073-6.

Morris JA (1999). The kinetics of epithelial cell generation: its relevance to cancer and ageing. $J$ Theoret Biol 199: 87-95.

Parkin DM, Muir CS, Whelan SL, et al. (1992). Cancer Incidence in Five Continents, Vol VI. IARC, Lyon.

Peeters PHM, Verbeek ALM, Krol A, Matthyssen MMM, DeWaard F (1994). Age at menarche and breast cancer risk in nulliparous women. Breast Cancer Res Treat 33: 55-61.

Potten CS, Loeffler M (1990). Stem cells: attributes, cycles, spirals, pitfalls and uncertainties. Lessons for and from the crypt. Development 110: 1001-19.

Trichopoulos D (1990). Hypothesis: does breast cancer originate in utero? Lancet 335: 939-40.

UK Testicular Cancer Study Group (1994). Social, behavioural and medical factors in the aetiology of testicular cancer: results from the UK study. Br J Cancer 70: 513-20.

Vogelstein B, Fearon ER, Hamilton, et al. (1998). Genetic alterations during colorectal tumour development. $N$ Engl J Med 319: 525-32.

\section{Appendix}

Let $m$ equal the mutation rate per gene per cell division, $y$ is the mutation rate per gene per annum in the absence of cell division and $n$ is the number of specific independent mutations required for cancer. The probability that a single stem cell becomes malignant after $w$ generations and $z$ years is (Morris, 1991):

$$
(w m+z y)^{n}
$$

Probabilities are added in the above function as $m$ and $y$ are much less than 1 ( $m$ has values between $10^{-7}$ and $10^{-6}, y$ is closer to $10^{-8}$ ). If there are $b$ cell generations between the stem cell and mature epithelial cells the probability that a mature epithelial cell has acquired the necessary mutations for malignancy is:

$$
([w+b] m+z y)^{n}
$$

If stem cells are destroyed by trauma it is possible that stem cell progeny take over the stem cell role, in which case the risk of cancer will rise. In the case of the conventional model the increase is small. If $m=10^{-6}, y=10^{-8}, w=5000, b=10, z=70$ and $n=4$, which are all reasonable values derived from published literature (Morris, 1990, 1991; Potten and Loeffler, 1990; Vogelstein et al., 1998), the increased cancer risk is:

$$
\frac{([w+b] m+z y)^{n}}{(w m+z y)^{n}}=1.008
$$

In the hierarchical model the senior stem cell is approximately 35 generations from the zygote and mature epithelial cells are closer to 55 generations from the zygote. The above ratio is now:

$$
\frac{(55 m+z y)^{n}}{(35 m+z y)^{n}}=5.9
$$

Thus physical trauma in the hierarchical model can in theory cause a marked increase in the incidence of cancer.

The situation in the case of the hierarchical model could be more extreme. If the stem cell hierarchy is disrupted there is no guarantee that the new stem cell will form a new hierarchy. It is possible that the new stem cell will undergo regular cycling as in the conventional model. In which case if it cycles once per week for 2 years the risk of cancer after 2 years in the damaged area compared with what it would have been if no damage had occurred is:

$$
\frac{(159 m+z y)^{n}}{(35 m+z y)^{n}}=399
$$

Trauma only affects part of the breast so that the increase in risk to the patient is less than the increase in the damaged area. The above considerations indicate, however, that it is biologically plausible for trauma to increase the risk to the patient by the amount noted in this study, i.e. odds ratio of 3.3 (95\% CI 1.3-10.8).

It is also worth noting that the relative risk of cancer in a stem cell at age 70 years in the conventional model compared with the hierarchical model is:

$$
\frac{(5000 m+z y)^{n}}{(35 m+z y)^{n}}=10^{8}
$$

This result alone provides a strong argument in favour of the model based on a stem cell hierarchy. 